\title{
Chiral route to spontaneous entanglement generation
}

\author{
Carlos Gonzalez-Ballestero, ${ }^{1, *}$ Alejandro Gonzalez-Tudela, ${ }^{2}$ Francisco J. Garcia-Vidal, ${ }^{1,3}$ and Esteban Moreno ${ }^{1}$ \\ ${ }^{1}$ Departamento de Física Teórica de la Materia Condensada and Condensed Matter Physics Center (IFIMAC), \\ Universidad Autónoma de Madrid, E-28049 Madrid, Spain \\ ${ }^{2}$ Max-Planck-Institut für Quantenoptik Hans-Kopfermann-Strasse 1, 85748 Garching, Germany \\ ${ }^{3}$ Donostia International Physics Center (DIPC), E-20018 Donostia/San Sebastian, Spain
}

(Received 18 July 2015; revised manuscript received 17 September 2015; published 6 October 2015)

\begin{abstract}
We study the spontaneous entanglement generation between two qubits chirally coupled to a waveguide. The maximum achievable concurrence is demonstrated to increase by a factor of $4 / e \sim 1.5$ as compared to the nonchiral coupling situation. The proposed entanglement scheme is shown to be robust against variation of the qubit properties such as detuning and separation, which are critical in the nonchiral case. This result relaxes the restrictive requirements of the nonchiral situation, paving the way toward a realistic implementation. Our results demonstrate the potential of chiral waveguides for quantum entanglement protocols.
\end{abstract}

DOI: 10.1103/PhysRevB.92.155304

\section{INTRODUCTION}

Efficient quantum circuits are a very important ingredient for the development of quantum computing [1]. Usual implementations of these devices require platforms where information, usually in the form of photons, can be easily introduced and extracted [2]. Several systems based on waveguides have been proposed for quantum circuitry, from superconducting stripes [3] to dielectric [4,5], photonic crystal [6,7], or plasmonic waveguides [8]. In this context, the interaction between the guided photons and quantum emitters is critical for various processes, such as the creation of entangled states between the qubits. Spontaneous entanglement generation in waveguide setups has already been predicted $[9,10]$. Many other interesting phenomena, such as mesoscopic entanglement [11], long-distance quantum beats [12], or the formation of sub- and superradiant states [13], show that waveguides are excellent platforms for quantum-information processing.

Recently, systems of emitters chirally coupled to waveguides have attracted a lot of attention both theoretically and experimentally [14-16]. In these configurations, an adequate engineering of the waveguide can be used to break the emission symmetry of the qubits, channeling the emitted photons preferentially into one of the two directions of the waveguide. Chirality in emitter-waveguide coupling is a general effect associated with the so-called spin-orbit interaction of light [17]. In addition to theoretical studies, many experiments have reported chiral emission with a very large degree of directionality, from nanoparticles and atomic ensembles in dielectric waveguides $[17,18]$ to quantum dots in nanobeams [19] and photonic crystals [20-22]. Among these setups, the latter turn out to be especially promising systems as they combine large directionalities of around $90 \%$ with high emitter-waveguide coupling fractions (up to $98 \%$ ). Consequently, they have been proposed as ideal platforms for implementation of quantum logical gates [22].

In this paper, we tackle the problem of spontaneous entanglement generation between two qubits chirally coupled to a waveguide. In the first part of this work, we present an analytical solution to the master equation describing the evolution

\footnotetext{
*carlos.ballestero@uam.es
}

PACS number(s): 42.50.Ex, 03.67.Bg, 42.50.Ct, 42.79.Gn

of the system state, showing how chirality allows for an enhancement up to $\sim 50 \%$ in the maximum generated entanglement as compared to the nonchiral case. In the second part of the paper, we present a more complete formalism in which nonMarkovian effects are explicitly accounted for by fully diagonalizing the Hamiltonian in the single-excitation subspace. We use this formalism to demonstrate the robustness of the entanglement generation scheme against the detuning between the qubits, the total coupling rate, and the qubit-qubit separation.

\section{MARKOVIAN APPROACH}

The system under study is depicted in Fig. 1. Two emitters 1 and 2, modeled as two-level systems of frequency $\omega_{0}$, are coupled to the propagating photonic modes of a waveguide. The emitters are separated by a distance $d=x_{2}-x_{1}$, and they are coupled to the right- and left-propagating photons through the energy constants $\gamma_{j R}$ and $\gamma_{j L}(j=1,2)$, respectively. In a chiral coupling scheme such as the one analyzed in this work, these constants are different $\left(\gamma_{j R} \neq \gamma_{j L}\right)$. Other deexcitation processes into free space or additional lossy modes are taken into account through the decay rates $\Gamma_{j}$. The three coupling constants of each qubit are used to define a usual figure of merit in waveguide systems, namely the coupling fraction or beta factor, given by $\beta_{j}=\left(\gamma_{j R}+\gamma_{j L}\right) /\left(\gamma_{j R}+\gamma_{j L}+\Gamma_{j}\right)$.

Our aim is to analyze spontaneous entanglement generation when qubit 1 (the qubit on the left) is initially excited. We start by solving the system dynamics, obtaining the time evolution of the reduced density matrix of the two-qubit subsystem, $\rho$. In the first part of this work, we will follow a usual approach undertaken in quantum optics, in which the problem is simplified by tracing out the photonic degrees of freedom under the so-called Markov approximation [23]. In this situation, the only dynamical variable is the density matrix $\rho$, whose evolution is governed by the following general master equation $(\hbar=1)$ :

$$
\begin{aligned}
\dot{\rho}= & -i[H, \rho]+\sum_{j=1,2} \gamma_{j} \mathcal{L}_{\sigma_{j}, \sigma_{j}}[\rho] \\
& +\sqrt{\gamma_{2 R} \gamma_{1 R}} \mathcal{L}_{\sigma_{2}, \sigma_{1}}[\rho]+\sqrt{\gamma_{2 L} \gamma_{1 L}} \mathcal{L}_{\sigma_{1}, \sigma_{2}}[\rho],
\end{aligned}
$$

where we define $\gamma_{j} \equiv\left(\gamma_{j R}+\gamma_{j L}\right) / 2$. The bare Hamiltonian of the system is given by $H=\omega_{0}\left(\sigma_{1}^{\dagger} \sigma_{1}+\sigma_{2}^{\dagger} \sigma_{2}\right)$, where $\sigma_{j}$ is 


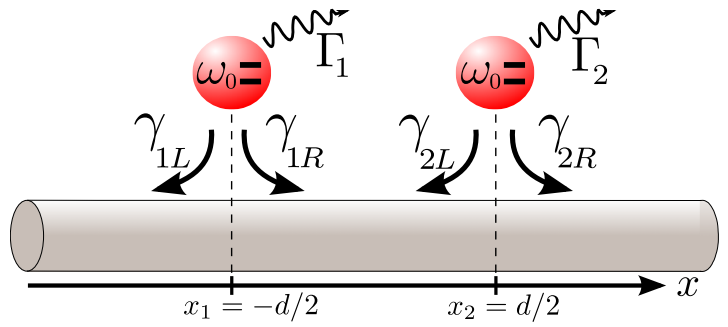

FIG. 1. (Color online) System under study. Two qubits of frequency $\omega_{0}$ and separated by a distance $d$ are placed in the vicinities of a waveguide. The energies $\gamma_{j \alpha}$ quantify the chiral coupling of qubit $j$ to the photonic propagating mode $\alpha(=L, R)$. In the same fashion, the decay rate into the $3 \mathrm{D}$ environment and other lossy modes is described by a coupling constant $\Gamma_{j}$.

the annihilation operator of qubit $j$. The generalized Lindblad superoperators, $\mathcal{L}_{\sigma_{a}, \sigma_{b}}$, are expressed as

$$
\mathcal{L}_{\sigma_{a}, \sigma_{b}}[\rho]=\left(e^{-i 2 \pi D_{a b}}\left[\sigma_{a}, \rho \sigma_{b}^{\dagger}\right]-e^{i 2 \pi D_{a b}}\left[\sigma_{a}^{\dagger}, \sigma_{b} \rho\right]\right),
$$

where $D_{a b} \equiv\left|x_{a}-x_{b}\right| / \lambda_{0}, \lambda_{0}=2 \pi v_{g} / \omega_{0}$ is the emission wavelength of the qubits, and $v_{g}$ is the group velocity of the guided photons. These terms are employed to describe both the decay of the qubits into the waveguide modes $(a=b)$ and the waveguide-mediated interaction between emitters $(a \neq b)$. In the latter case, the real (imaginary) part of $\mathcal{L}_{\sigma_{a}, \sigma_{b}}$ in Eq. (2) leads to an incoherent (coherent) coupling between the qubits $[10,24]$. Note that Eq. (1) is particular of chiral configurations [15], and it is reduced to its more common form in the case $\gamma_{j R}=\gamma_{j L}$. To focus on the fundamental aspects of the chiral system, we will first particularize our study to the lossless case (i.e., $\beta_{j}=1$ ), including the losses in the second part of the work.

Combining Eq. (1) with the particular initial conditions of our problem, $\rho(0)=\sigma_{1}^{\dagger}|0\rangle\langle 0| \sigma_{1}$, and expressing $\rho$ in the usual basis $\left\{|0\rangle,|1\rangle \equiv \sigma_{1}^{\dagger}|0\rangle,|2\rangle \equiv \sigma_{2}^{\dagger}|0\rangle,|3\rangle \equiv \sigma_{1}^{\dagger} \sigma_{2}^{\dagger}|0\rangle\right\}$, the only nonzero elements of the density matrix are the populations $\rho_{00}$, $\rho_{11}, \rho_{22}$, and the coherence $\rho_{12}$. Three of these quantities are coupled through the following system of differential equations:

$$
\begin{gathered}
\dot{\rho}_{11}=-2 \gamma_{1} \rho_{11}-\sqrt{\gamma_{1 L} \gamma_{2 L}}\left(e^{i 2 \pi \tilde{d}} \rho_{21}+e^{-i 2 \pi \tilde{d}} \rho_{12}\right), \\
\dot{\rho}_{22}=-2 \gamma_{2} \rho_{22}-\sqrt{\gamma_{1 R} \gamma_{2 R}}\left(e^{i 2 \pi \tilde{d}} \rho_{12}+e^{-i 2 \pi \tilde{d}} \rho_{21}\right), \\
\dot{\rho}_{12}=-\left(\gamma_{1}+\gamma_{2}\right) \rho_{12}-\sqrt{\gamma_{1 R} \gamma_{2 R}} \rho_{11} e^{-i 2 \pi \tilde{d}} \\
-\sqrt{\gamma_{1 L} \gamma_{2 L}} \rho_{22} e^{i 2 \pi \tilde{d}}
\end{gathered}
$$

with the normalized distance $\tilde{d}=d / \lambda_{0}$. Once these equations are solved, we can compute the qubit-qubit entanglement, which we quantify with the Wootters concurrence $C$ [25], a widely used measure thanks to its simple calculation and its intuitive bounds. Indeed, the concurrence ranges from 0 for nonentangled states to 1 for maximally entangled configurations. A straightforward calculation demonstrates that in this case, $C=2\left|\rho_{12}\right|$. An analytical solution of Eq. (1) can be obtained when the two qubits are equally coupled, i.e., $\gamma_{1}=\gamma_{2}=\gamma$. The following expression for the concurrence is
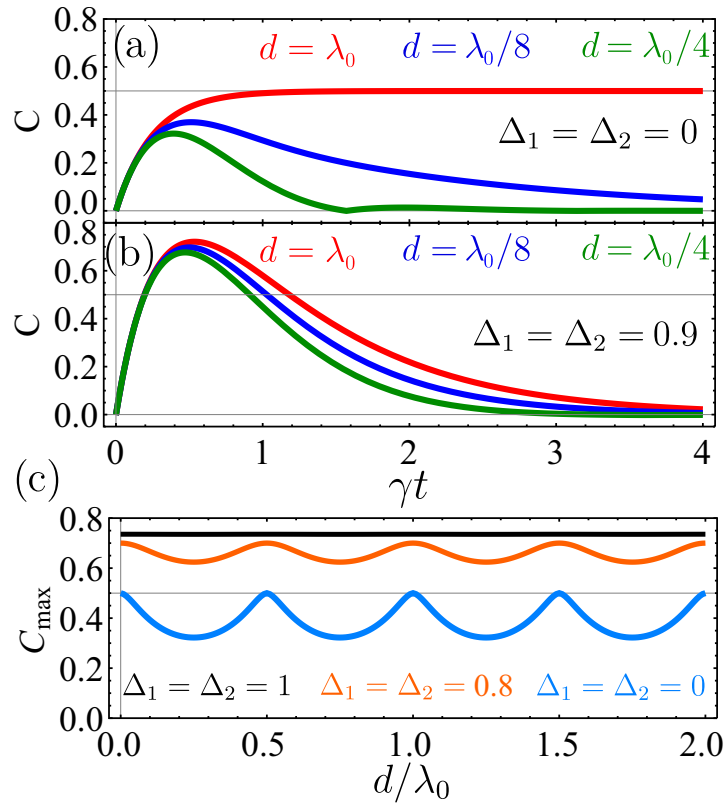

FIG. 2. (Color online) Time evolution of the concurrence, Eq. (6), for different qubit-qubit separations $d$, in the nonchiral (a) and chiral (b) cases. (c) Dependence of $C_{\max }$ on the separation between the qubits, $d$. The condition $\Delta_{1}=\Delta_{2}$ is chosen because it optimizes the maximum achievable concurrence $C_{\max }$ (see the main text). In all three panels, the ideal case $\beta_{j}=1$ is considered.

obtained:

$$
\begin{aligned}
C^{2}(t)= & \sqrt{\frac{\left(1+\Delta_{1}\right)\left(1+\Delta_{2}\right)}{\left(1-\Delta_{1}\right)\left(1-\Delta_{2}\right)}} e^{-4 \gamma t} \\
& \times\left(\sin ^{2}[2 q \gamma t \sin (2 \pi \tilde{d})]+\sinh ^{2}[2 q \gamma t \cos (2 \pi \tilde{d})]\right),
\end{aligned}
$$

where $q \equiv\left(1-\Delta_{1}^{2}\right)^{1 / 4}\left(1-\Delta_{2}^{2}\right)^{1 / 4}$, and we have introduced the directionality of qubit $j$ as the adimensional ratio $\Delta_{j}=$ $\left(\gamma_{j R}-\gamma_{j L}\right) /\left(\gamma_{j R}+\gamma_{j L}\right)$. The two terms in the second line of Eq. (6) represent, respectively, the coherent and incoherent contributions of the qubit-qubit interaction.

To have a clear view of the advantages brought about by chirality, let us first analyze the nonchiral case, $\Delta_{j}=0$. The time evolution of the concurrence in this situation is displayed in Fig. 2(a). Regardless of the separation between the qubits, the concurrence is 0 for $t=0$ since the initial state is separable, and it grows up to a maximum value $C(t)>0$. This spontaneous entanglement generation is caused by the imbalance between the decay rates of the two entangled eigenstates of the Liouvillian (namely, the symmetric or antisymmetric combination of excitations). As the system evolves in time, the superradiant component rapidly decays while the subradiant one remains populated for a longer period [10]. For $t \rightarrow \infty$, the behavior of the concurrence depends on the separation $d$. For almost all separations, the concurrence decreases to zero following the decay of the longlived subradiant state. However, when the qubits are identical and for very specific separations, $2 \tilde{d}=0,1,2, \ldots$, the behavior is different, as the concurrence has an infinite lifetime. In this particular situation, the subradiant state becomes a dark 
state, which is totally uncoupled from the waveguide. As a consequence, the concurrence does not decay, and it reaches its maximum achievable value $C=0.5$, bounded by the $50 \%$ overlap between the initial state and the dark state. The appearance of an uncoupled state for this particular separation is caused by a Fabry-Pérot-like resonance between the qubits, where a photon of energy $\omega_{0}$ can be trapped forming a standing wave [26]. The presence of this localized photon is linked to the fact that the transmittance of one qubit is strictly zero for an incoming resonant photon [27]. Note that, in a realistic case $(\beta<1)$, the concurrence always decays with time [10].

When the coupling is chiral, on the other hand, a straightforward calculation demonstrates that the above-mentioned standing wave does not appear (see the Appendix for details), as the chirality effectively couples the right- and left-propagating modes, and, consequently, the single-qubit transmittance never vanishes. Thus, the appearance of a dark state is prevented by chirality. As a consequence, the dependence of the concurrence on the separation $d$ becomes less critical, as Fig. 2(b) shows. Moreover, the chiral coupling also modifies the mechanism responsible for the entanglement generation, which now does not rely only on the incoherent part of the interaction. Indeed, in the chiral case, the Liouvillian contains an extra Hamiltonian part [15] that induces an additional, coherent transfer of excitations between the qubits. As a result of this supplementary interaction, the concurrence is no longer bounded by the overlap between the initial state and the dark state, and it is then able to reach higher values than in the nonchiral situation [see Fig. 2(b)]. The maximum concurrence achieved during the time evolution, $C_{\max }$, is displayed in Fig. 2(c) as a function of $d$. Clearly, the maximally chiral configuration $\Delta_{j}=1$ optimizes the entanglement generation scheme, as $C_{\max }$ reaches a maximum value that, additionally, is independent of $d$. Chirality thus represents an advantage toward a realistic implementation, as it can overcome the critical dependence on the separation in nonchiral configurations.

In spite of the weak dependence with the qubit-qubit separation, there exist nevertheless specific separations that are optimum for the entanglement generation. As Fig. 2(c) shows, the concurrence $C_{\max }$ is maximized when the distance between the qubits is $2 \tilde{d}=0,1,2, \ldots$. For these separations, an analytical expression can be extracted from Eq. (6),

$$
C_{\max }^{2}=\frac{\left(1+\Delta_{1}\right)\left(1+\Delta_{2}\right)}{1-q^{2}}\left(\frac{1-q}{1+q}\right)^{\frac{1}{q}} .
$$

To understand the dependence of $C_{\max }$ with the directionalities, which is shown in Fig. 3, it is important to bear in mind that initially only qubit 1 is excited. For $\Delta_{j}=-1$, the concurrence is strictly zero during all the time evolution, as the qubit $j$ is coupled only to left-propagating modes and thus is not able to interact with its partner. On the other hand, any values of the directionalities in the region $\Delta_{1}, \Delta_{2}>0$ result in an enhancement of $C_{\max }$ with respect to the nonchiral case. Moreover, when both qubits are maximally coupled to right-propagating modes, i.e., $\Delta_{1}=\Delta_{2}=1$, the maximum entanglement rises up to a very large value, which can be extracted from Eq. (7) as $\lim _{q \rightarrow 0} C_{\max }=2 / e \sim 0.73$. This is a significant result, as it shows that the maximum achievable

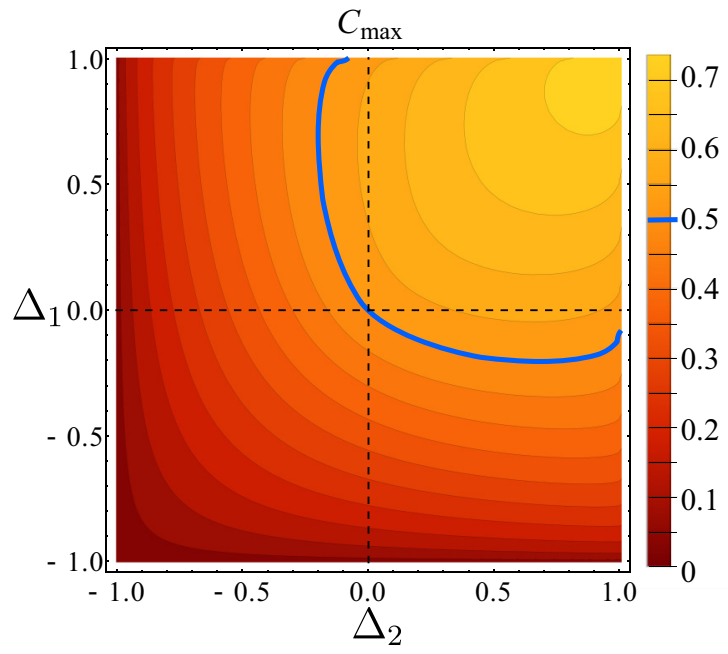

FIG. 3. (Color online) Maximum achievable concurrence in the ideal case $(\beta=1)$, vs the directionalities of each qubit, $\Delta_{1}, \Delta_{2}$, for the left qubit initially excited, and a separation $d=\lambda_{0}$. The blue line displays the nonchiral value $C_{\max }=0.5$.

concurrence can be enhanced by a factor of $\sim 50 \%$ with respect to the nonchiral coupling scheme.

\section{NON-MARKOVIAN REGIME}

We have seen that playing upon chirality, it is possible both to reach higher values of the maximum concurrence, $C_{\max }$, and to reduce the sensitivity to the qubit separation, $d$. However, for large separations or coupling rates, $\gamma d \gg v_{g}$, it has been reported that non-Markovian effects arise in waveguide systems [26,28-30]. Such effects introduce additional retardation that cannot be described with the currently used master equation formalism. Hence, we now reformulate the problem to properly assess the robustness of the proposed protocol. We employ a more complete approach by diagonalizing the full Hamiltonian of the system in the single excitation subspace, as detailed in the Appendix. Unless stated otherwise, we will assume equally coupled qubits, i.e., $\gamma_{1 R}=\gamma_{2 R}$ and $\gamma_{1 L}=\gamma_{2 L}$ and large directionalities $\left(\Delta_{j}=0.90\right)$ in order to stay close to the optimum configuration. We also include explicitly the lossy modes in this part of our work by fixing the decay rates $\Gamma_{j}$ such that $\beta_{j}=0.98$. Note that the chosen values for both the directionalities and the beta factors have been experimentally reported [22].

We employ the above-mentioned formalism to explore the robustness of the scheme against variation of three parameters, namely the detuning between the frequencies of the emitters, the total coupling to the waveguide modes, $\gamma$, and the separation $d$. In Fig. 4(a), the effect of detuning between the qubits is studied. For the corresponding calculations, the frequencies of both emitters are modified according to $\omega_{1}=\omega_{0}+\delta / 2$ and $\omega_{2}=\omega_{0}-\delta / 2$. Physically, we are shifting away the emission spectrum of qubit 1 from the absorption spectrum of qubit 2 , keeping their linewidth constant. It is known that a strong overlap between both the spectral distribution of the photon emitted by qubit 1 , and the absorption spectrum of qubit 2 , is key for entanglement generation [31]. Hence, the concurrence 

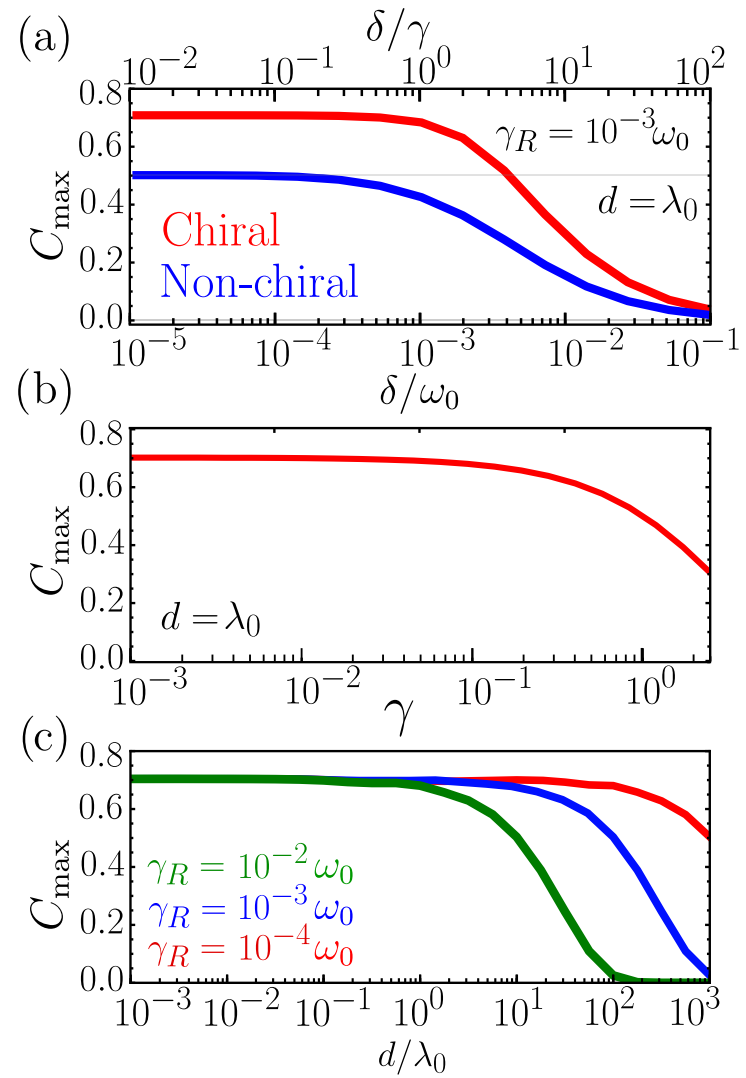

FIG. 4. (Color online) Maximum concurrence $C_{\max }$ as a function of the various system parameters $\left(\Delta_{j}=0.9, \beta_{j}=0.98\right)$. (a) Effect of the detuning between the transition frequencies of the qubits, $\delta$. (b) Dependence on the total coupling to the waveguide, $\gamma$. (c) Dependence on the qubit-qubit separation, $d$, for different values of $\gamma$. All the coupling constants are normalized to $\omega_{0}$.

naturally decreases for large values of $\delta$. The detuning relative to $\omega_{0}$ seems to be the most critical parameter as a change of $\sim 0.5 \%$ in the frequency of the qubits is enough to reduce $C_{\max }$ below 0.5 . However, the robustness against detuning is considerably large with respect to the qubits linewidth, $\gamma$. Indeed, whereas in the nonchiral case the concurrence is independent of the detuning for $\delta \lesssim 0.2 \gamma$, for chiral couplings this range is increased by a factor of $\sim 5$. Additionally, in the chiral case, concurrences of $C_{\max }=0.5$ are possible for detunings as large as $\delta \sim 5 \gamma$. This is a crucial advantage with respect to nonchiral systems, especially for quantum emitters with a very narrow linewidth such as quantum dots.

The variation of $C_{\max }$ with the total qubit-waveguide coupling is displayed in Fig. 4(b). For low values of $\gamma$, the concurrence is close to its theoretical maximum $\left(C_{\max } \sim 0.7\right)$ due to the large chosen directionalities. When $\gamma$ is increased, the photonic wave packet emitted by qubit 1 becomes narrower in space [31], and, eventually, its width becomes smaller than or comparable to the qubit-qubit separation $d$. As a result, qubit 1 significantly decays before the photon reaches qubit 2 , and, at any given time, at least one of the emitters is fairly depopulated. Thus, the concurrence $C=2\left|\rho_{12}\right| \equiv 2 \sqrt{\rho_{11} \rho_{22}}$ decreases for large values of $\gamma$. This effect has been studied in detail in nonchiral configurations [26]. Note that, nevertheless, even for couplings as large as $\gamma \approx 0.1 \omega_{0}$, the concurrence remains above 0.6 . This result shows that chirality allows for a high level of concurrence not only in the optical regime, but also in systems in which much larger couplings arise such as microwave striplines.

Finally, the variation of the concurrence with the qubit-qubit separation $d$ is shown in Fig. 4(c). While in the nonchiral case a maximum concurrence of 0.5 was obtained only for particular values of $d$, for chiral couplings the entanglement generation scheme is shown to be robust for a wide range of separations. For large distances, there is a decay in the concurrence, which responds to the same mechanism discussed above. In this case, the spatial extension of the emitted photon is made smaller than the separation $d$ by directly increasing the qubit separation instead of the coupling $\gamma$. Interestingly, for qubit-waveguide couplings in the optical regime $\left(\gamma \lesssim 10^{-4} \omega_{0}\right)$, the entanglement generation scheme is extremely robust with respect to the distance $d$, allowing for concurrences above 0.6 for very large separations, e.g., around $60 \mu \mathrm{m}$ at $\omega_{0} \sim 2 \mathrm{eV}$. The separation between the qubits is thus not a critical parameter anymore, allowing for a much easier implementation of this entanglement protocol.

\section{CONCLUSION}

In conclusion, the phenomenon of spontaneous generation of entanglement between two qubits chirally coupled to a waveguide has been analyzed in detail. We show that even the slightest directionalities in the couplings may improve the maximum achievable entanglement, as compared to nonchiral systems. Moreover, we identify the optimal directionalities and demonstrate a very significant enhancement of the maximum entanglement. This entangling scheme displays a fairly weak dependence on the relevant parameters, which highlights the robustness of the protocol. In particular, when compared to the nonchiral case, the influence of the qubit-qubit separation is reduced, which constitutes an important advantage for the feasibility of an experimental implementation. In addition to the interest of our specific results, our work positions entanglement as one further reason for the exploration of chiral waveguide QED.

\section{ACKNOWLEDGMENTS}

C.G.B. acknowledges the Spanish MECD (FPU13/01225 fellowship). A.G.T. acknowledges financial support from the Marie Curie Intra European Fellowship NanoQuIS (625955). C.G.B. and F.J.G.V. acknowledge the European Research Council (ERC-2011-AdG Proposal No. 290981). E.M., F.J.G.V., and C.G.B. acknowledge the Spanish MINECO (Grant No. MAT2014-53432-C5-5-R).

\section{APPENDIX: DIAGONALIZATION OF THE HAMILTONIAN}

We make use of the real-space formalism, which is usually employed in low-excitation problems in waveguide QED [32]. The Hamiltonian of the system is $H=H_{\mathrm{qb}}+H_{\mathrm{wg}}+H_{I}$, where the two first terms correspond to the energy of the qubits and the waveguide, respectively, and $H_{I}$ is the interaction term. 
They are given by $(\hbar=1)$

$$
\begin{gathered}
H_{\mathrm{qb}}=\sum_{j} \omega_{j} \sigma_{j}^{\dagger} \sigma_{j}, \\
H_{\mathrm{wg}}=i v_{g} \int d x\left[c_{L}^{\dagger}(x) \partial_{x} c_{L}(x)-c_{R}^{\dagger}(x) \partial_{x} c_{R}(x)\right], \\
H_{I}=\sum_{j=1}^{2} \sum_{\alpha=R, L} \int d x \delta\left(x-x_{j}\right)\left[V_{j \alpha} c_{\alpha}^{\dagger}(x) \sigma_{j}+\text { H.c. }\right] .
\end{gathered}
$$

In the above equations, $\Omega_{j}$ is the transition frequency of qubit $j$, and $v_{g}$ is the group velocity of the guided modes, whose dispersion is considered linear. The constants $V_{j \alpha}$, assumed real for simplicity, are related to the coupling rates in the main text through $\gamma_{j \alpha}=V_{j \alpha}^{2} / v_{g}$. The operators $\sigma_{j}$ and $c_{\alpha}(x)$ destroy an excitation in qubit $j$ and an $\alpha$-propagating photon at position $x$, respectively. Finally, $x_{j}= \pm d / 2$ is the position of the emitter $j$ along the waveguide. Note that the losses $\Gamma_{j}$ are not accounted for in Eq. (A1), as we introduce them a posteriori.

To diagonalize the system Hamiltonian in the single excitation subspace, we assume an eigenstate of the form

$$
|\epsilon\rangle=\left(\sum_{j} \alpha_{j} \sigma_{j}^{\dagger}+\sum_{\alpha} \int d x \phi_{\alpha}(x) c_{\alpha}^{\dagger}(x)\right)|0\rangle,
$$

and we solve the time-independent Schrödinger equation, $H|\epsilon\rangle=\epsilon|\epsilon\rangle$, to obtain the coefficients $\left\{\alpha_{j}, \phi_{\alpha}(x)\right\}$. Following the usual approach, we make a plane-wave ansatz for the wave functions $\phi_{\alpha}(x)$, i.e.,

$$
\begin{aligned}
& \phi_{R}(x)=e^{i \epsilon x / v_{g}} \begin{cases}A & \text { for } \quad x<-d / 2, \\
B & \text { for }-d / 2<x<d / 2, \\
C & \text { for } \quad d / 2<x,\end{cases} \\
& \phi_{L}(x)=e^{-i \epsilon x / v_{g}} \begin{cases}D & \text { for } \quad x<-d / 2, \\
E & \text { for } \quad-d / 2<x<d / 2, \\
F & \text { for } \quad d / 2<x,\end{cases}
\end{aligned}
$$

which reduces the problem to an algebraic system of equations [32]. For each energy $\epsilon$, we can naturally find two linearly independent eigenstates, corresponding to the scattering of photons coming from either $x=-\infty$ or $\infty$. These states, labeled $\left|\epsilon_{+}\right\rangle$and $\left|\epsilon_{-}\right\rangle$, are obtained by setting $\{A=1, F=0\}$ and $\{F=1, A=0\}$ in Eqs. (A5) and (A6), respectively. The eigenstates $\left|\epsilon_{ \pm}\right\rangle$, whose degeneracy is inherited from that of the right- or left-propagating mode of a bare waveguide, have been called scattering eigenstates in the literature.

The scattering solutions of the Schrödinger equation have been reported to not always form a complete basis, as localized resonances may arise [26]. We thus need to check our equations for orthogonal solutions, i.e., $A=F=0$. A simple calculation shows that the necessary conditions for a localized eigenstate are $\gamma_{1 R}=\gamma_{1 L}$ and $\gamma_{2 R}=\gamma_{2 L}$. We conclude that, in the chiral case, the basis $\left\{\left|\epsilon_{ \pm}\right\rangle\right\}$is complete, as no localized resonances appear.

With the eigenstates at hand, we are able to introduce the qubit losses, $\Gamma_{j}$. As localized eigenstates are not present in the chiral case, the effect of the lossy modes is easily accounted for. Indeed, it has been shown in Ref. [26] that the effect of the external decay of the qubits over the scattering eigenstates can be fully reproduced by adding an imaginary part to the frequency of the emitters, $\omega_{j} \rightarrow \omega_{j}-i \Gamma_{j} / 2$.

The final step for obtaining the system dynamics is to construct the time evolution operator, $U(t)$. If the coupling is chiral, this task is not as straightforward as in previous works, as the two scattering branches are not orthogonal. The operator $U(t)$ takes now a more complicated form:

$$
U(t)=\frac{1}{2 \pi v_{g}} \sum_{i, j= \pm} \int_{-\infty}^{\infty} d \epsilon e^{-i \epsilon t}\left|\epsilon_{i}\right\rangle\left(S^{-1}\right)_{i j}\left\langle\epsilon_{j}\right|,
$$

where we define the overlap matrix $S$ as

$$
S_{i j}=\lim _{L \rightarrow \infty} \frac{\left\langle\epsilon_{i} \mid \epsilon_{j}\right\rangle}{L} .
$$

Using (A7), we can numerically obtain the evolution of any initial state through $|\psi(t)\rangle=U(t)|\psi(0)\rangle$. Finally, it can be checked that $U(0)=1$, which certifies the completeness of the basis $\left\{\left|\epsilon_{ \pm}\right\rangle\right\}$.
[1] H. J. Kimble, The quantum internet, Nature (London) 453, 1023 (2008).

[2] J. L. O’Brien, Optical quantum computing, Science 318, 1567 (2007).

[3] T. Niemczyk, F. Deppe, H. Huebl, E. P. Menzel, F. Hocke, M. J. Schwarz, J. J. Garcia-Ripoll, D. Zueco, T. Hummer, E. Solano, A. Marx, and R. Gross, Circuit quantum electrodynamics in the ultrastrong-coupling regime, Nat. Phys. 6, 772 (2010).

[4] Q. Quan, I. Bulu, and M. Lončar, Broadband waveguide qed system on a chip, Phys. Rev. A 80, 011810 (2009).

[5] J.-B. Béguin, E. M. Bookjans, S. L. Christensen, H. L. Sørensen, J. H. Müller, E. S. Polzik, and J. Appel, Generation and Detection of a Sub-Poissonian Atom Number Distribution in a One-Dimensional Optical Lattice, Phys. Rev. Lett. 113, 263603 (2014).

[6] C. W. Wong, J. Gao, J. F. McMillan, F. W. Sun, and R. Bose, Quantum information processing through quantum dots in slow-light photonic crystal waveguides, Photon. Nanostruct.Fundament. Appl. 7, 47 (2009).

[7] A. Goban, C. L. Hung, S. P. Yu, J. D. Hood, J. A. Muniz, J. H. Lee, M. J. Martin, A. C. McClung, K. S. Choi, D. E. Chang, O. Painter, and H. J. Kimble, Atom-light interaction in photonic crystals, Nat. Commun. 5, 3808 (2014).

[8] A. V. Akimov, A. Mukherjee, C. L. Yu, D. E. Chang, A. S. Zibrov, P. R. Hemmer, H. Park, and M. D. Lukin, Generation of single optical plasmons in metallic nanowires coupled to quantum dots, Nature (London) 450, 402 (2007).

[9] D. Dzsotjan, A. S. Sørensen, and M. Fleischhauer, Quantum emitters coupled to surface plasmons of a nanowire: A Green's function approach, Phys. Rev. B 82, 075427 (2010).

[10] A. Gonzalez-Tudela, D. Martin-Cano, E. Moreno, L. MartinMoreno, C. Tejedor, and F. J. Garcia-Vidal, Entanglement of Two Qubits Mediated by One-Dimensional Plasmonic Waveguides, Phys. Rev. Lett. 106, 020501 (2011). 
[11] A. González-Tudela and D. Porras, Mesoscopic Entanglement Induced by Spontaneous Emission in Solid-State Quantum Optics, Phys. Rev. Lett. 110, 080502 (2013).

[12] H. Zheng and H. U. Baranger, Persistent Quantum Beats and Long-Distance Entanglement from Waveguide-Mediated Interactions, Phys. Rev. Lett. 110, 113601 (2013).

[13] A. F. van Loo, A. Fedorov, K. Lalumière, B. C. Sanders, A. Blais, and A. Wallraff, Photon-mediated interactions between distant artificial atoms, Science 342, 1494 (2013).

[14] T. Ramos, H. Pichler, A. J. Daley, and P. Zoller, Quantum Spin Dimers from Chiral Dissipation in Cold-Atom Chains, Phys. Rev. Lett. 113, 237203 (2014).

[15] H. Pichler, T. Ramos, A. J. Daley, and P. Zoller, Quantum optics of chiral spin networks, Phys. Rev. A 91, 042116 (2015).

[16] F. Le Kien and A. Rauschenbeutel, Anisotropy in scattering of light from an atom into the guided modes of a nanofiber, Phys. Rev. A 90, 023805 (2014).

[17] J. Petersen, J. Volz, and A. Rauschenbeutel, Chiral nanophotonic waveguide interface based on spin-orbit interaction of light, Science 346, 67 (2014).

[18] R. Mitsch, C. Sayrin, B. Albrecht, P. Schneeweiss, and A. Rauschenbeutel, Quantum state-controlled directional spontaneous emission of photons into a nanophotonic waveguide, Nat. Commun. 5, 5713 (2014).

[19] R. J. Coles, D. M. Price, J. E. Dixon, B. Royall, E. Clarke, A. M. Fox, P. Kok, M. S. Skolnick, and M. N. Makhonin, Chirality of nanophotonic waveguide with embedded quantum emitter for unidirectional spin transfer, arXiv:1506.02266.

[20] A. B. Young, A. Thijssen, D. M. Beggs, P. Androvitsaneas, L. Kuipers, J. G. Rarity, S. Hughes, and R. Oulton, Polarization engineering in photonic crystal waveguides for spin-photon entanglers, arXiv:1406.0714.

[21] B. le Feber, N. Rotenberg, and L. Kuipers, Nanophotonic control of circular dipole emission, Nat. Commun. 6, 6695 (2015).
[22] I. Söllner, S. Mahmoodian, S. Lindskov Hansen, L. Midolo, A. Javadi, G. Kiršanske, T. Pregnolato, H. El-Ella, E. H. Lee, J. D. Song, S. Stobbe, and P. Lodahl, Deterministic photon-emitter coupling in chiral photonic circuits, Nature Nanotechnology $\mathbf{1 0}$, 775 (2015).

[23] Quantum Statistical Properties of Radiation, edited by W. H. Louisell (Wiley, New York, 1973).

[24] R. Tanaś and Z. Ficek, Entangling two atoms via spontaneous emission, J. Opt. B 6, S90 (2004).

[25] W. K. Wootters, Entanglement of Formation of an Arbitrary State of Two Qubits, Phys. Rev. Lett. 80, 2245 (1998).

[26] C. Gonzalez-Ballestero, F. J. Garcia-Vidal, and E. Moreno, NonMarkovian effects in waveguide-mediated entanglement, New J. Phys. 15, 073015 (2013).

[27] J. T. Shen and S. Fan, Coherent photon transport from spontaneous emission in one-dimensional waveguides, Opt. Lett. 30, 2001 (2005).

[28] U. Dorner and P. Zoller, Laser-driven atoms in half-cavities, Phys. Rev. A 66, 023816 (2002).

[29] T. Tufarelli, M. S. Kim, and F. Ciccarello, Non-Markovianity of a quantum emitter in front of a mirror, Phys. Rev. A 90, 012113 (2014).

[30] Y.-L. L. Fang and H. U. Baranger, Waveguide qed: Power spectra and correlations of two photons scattered off multiple distant qubits and a mirror, Phys. Rev. A 91, 053845 (2015).

[31] C. Gonzalez-Ballestero, E. Moreno, and F. J. Garcia-Vidal, Generation, manipulation, and detection of two-qubit entanglement in waveguide qed, Phys. Rev. A 89, 042328 (2014).

[32] J.-T. Shen and S. Fan, Theory of single-photon transport in a single-mode waveguide. i. Coupling to a cavity containing a two-level atom, Phys. Rev. A 79, 023837 (2009). 